\section{Associação entre a cobertura de equipes de saúde bucal na saúde da família e o aumento na produção ambulatorial dos municípios brasileiros, 1999 e 2011}

\author{
Association between coverage by oral health \\ teams in the family health and the increase in \\ dental care output in Brazilian municipalities, \\ 1999 and 2011
}

\author{
Asociación entre cobertura de equipos de salud \\ bucal en salud de la familia y aumento de la \\ producción ambulatoria en municipios \\ brasileños, 1999 y 2011
}

\begin{abstract}
The aim of this study was to analyze the association between population coverage by oral health teams under the Family Health Strategy (FHS) and the difference in 1999 and 2011 in rates of use of public dental services in Brazilian municipalities. The sample included all 5,507 municipalities in both years. Data were used from government information systems to perform logistic regression for modeling the increase in procedure rates. By 2011, 85\% of Brazil's municipalities had oral health teams under the FHS and there had been an increase in infrastructure, human and financial resources. Dental care output increased 49.5\% from 1999 to 2011. Municipalities that incorporated more than 3 oral health teams per 10,000 inhabitants showed higher odds of increasing their rates of community procedures $(O R=1.61$, 95\%CI: 1.23-2.11), prophylactic procedures $(O R=$ 2.05, CI95\%: 1.56-2.69), restorations $(O R=2.07$, 95\%CI: 1.58-2.71), and extractions $(O R=1.53$, 95\%CI: 1.19-1.97) after adjusting for socio-demographic factors and variations in physical, human and financial resources. The incorporation of oral health teams into the FHS appears more effective for increasing indicators of the use of dental services.
\end{abstract}

Dental Health Services; Primary Health Care; Health Services Accessibility; Public Health Policy
Gabriel Trevizan Corrêa 1

Roger Keller Celeste 1

Analisar a associação entre a cobertura populacional de equipes de saúde bucal da Estratégia Saúde da Família (ESF) e a diferença nas taxas de uso de serviços odontológicos públicos nos municípios brasileiros em 1999 e 2011. A amos tra contou com 5.507 municípios nos 2 anos. Foram utilizados dados de sistemas de informação oficiais e se procedeu à regressão logística para modelar o incremento nas taxas de procedimentos. Observou-se que 85\% dos municípios possuíam equipes de saúde bucal da ESF em 2011 e houve aumentos nas taxas de recursos físicos, humanos e financeiros. A produção odontológica aumentou 49,5\% no período. Os municípios que incorporaram mais que 3 equipes de saúde bucal/10 mil habitantes tiveram mais chances de aumentar as taxas de procedimentos coletivos $(O R=1,61 ;$ IC95\%: 1,23-2,11), preventivos $(O R=2,05$; IC95\%: 1,56-2,69), restaurações $(O R=$ 2,07; IC95\%: 1,58-2,71) e extrações ( $O R=1,53$; IC95\%: 1,19-1,97), após controle por fatores sociodemográficos e relacionados à variação de recursos físicos, humanos e financeiros. A incorporação de equipes de saúde bucal à ESF parece mais efetiva para o aumento de indicadores de uso de serviços odontológicos.

Serviços de Saúde Bucal; Atenção Primária à Saúde; Acesso aos Serviços de Saúde; Políticas Públicas de Saúde 


\section{Introdução}

Desde os anos 1990, a atenção básica à saúde no Brasil vem sendo reorganizada pelo paradigma da Estratégia Saúde da Família, e tal modelo também foi adotado para os serviços de saúde bucal 1,2. Em 2001, as equipes de saúde bucal (ESB) passaram a ser incorporadas à Estratégia Saúde da Família (ESF), na proporção de uma ESB para cada duas equipes de saúde da família ${ }^{3}$, e em 2003 em qualquer proporção ${ }^{4}$. Assim, no ano de 2011, 4.847 municípios brasileiros já contavam com 21.038 dessas equipes. Além dessa expansão na oferta, a Política Nacional de Saúde Bucal 5 (PNSB) tem estimulado a qualificação do processo de atenção à saúde bucal, de forma a superar o modelo tradicional 6,7, caracterizado pelo preventivismo (ações coletivas e preventivas voltadas exclusivamente aos escolares) e ações clínicas mutiladoras. Diante disso, espera-se um resultado positivo também na utilização dos serviços odontológicos públicos no Brasil. Isso poderá ser mais bem estimado se for levado em conta que no período 1998-2003, o percentual de brasileiros que nunca consultaram o dentista declinou de $18,7 \%$ para $15,9 \%$ e posteriormente para 11,7\% em 2008 (Departamento de Informática do SUS. Sistema de Informações Ambulatoriais [SIH-SUS]. http://www.datasus.gov.br, acessado em Mai/2015), sendo essa redução atribuída em parte ao aumento da provisão de recursos financeiros e de serviços odontológicos 8 .

A saúde bucal na ESF representa a estratégia para a implantação de um novo modelo de Atenção Básica à saúde bucal, que se fundamenta no estabelecimento de territórios para o cuidado prioritário, controle das doenças bucais e seu impacto epidemiológico em médio e longo prazos ${ }^{7}$. Estudos sobre a influência da incorporação das ESB/ESF no uso dos serviços vêm sendo publicados nos últimos anos 9,10,11,12. Em nível nacional, após a introdução de ESB/ESF, houve um aumento de $6,9 \%$ na taxa total de procedimentos realizados e de $15,9 \%$ na taxa de procedimentos coletivos 9. Uma avaliação realizada no Município de Natal, Rio Grande do Norte, evidenciou maior uso de serviços odontológicos pelos habitantes das áreas de ESB/ESF (31,9\%) em comparação àqueles de áreas de atenção odontológica tradicional ou sem nenhuma cobertura populacional $(17,5 \%)$, porém menor do que o uso de moradores de áreas de ESF sem saúde bucal $(45,4 \%)$ 10. No Estado de Pernambuco, de 2002 a 2005, as ESB/ESF aumentaram 204,2\%, e a produção de ações básicas em odontologia cresceu $19,2 \%$ 11. É possível, então, que parte do referido aumento na utilização de serviços odontológicos, associado à presença das ESB/ESF, seja fruto desta reorientação no modelo de atenção, e não somente da expansão de infraestrutura.

Embora a provisão de fatores relacionados à infraestrutura de serviços de saúde seja considerada importante determinante da sua utilização 13 , tal relação não está claramente estabelecida. Estudos internacionais encontraram resultados contraditórios na relação entre uso de serviços odontológicos e o incremento da oferta 14,15,16,17,18. Por exemplo, apesar do aumento de acesso ao dentista, houve um declínio na utilização de serviços odontológicos por parte dos jovens brasileiros, nas capitais, durante o período de 1986 a 2002, possivelmente por melhorias nas condições de saúde bucal 18. Há evidências de que expansão ou contração de oferta não altera o uso dos serviços odontológicos, uma vez que ele parece sofrer forte influência de efeito de coorte de nascimento 17. Alguns estudos mostram que com a expansão de serviços gratuitos de atenção primária, as pessoas em menos desvantagem utilizam mais os serviços 19,20. Faz-se necessário, portanto, melhor entendimento sobre a real influência que as políticas de expansão e qualificação da oferta de serviços de saúde bucal exercem sobre sua utilização. O modelo teórico utilizado neste estudo foi baseado na abordagem metodológica de avaliação de serviços de saúde de Donabedian 21,22. Sendo assim, o objetivo foi descrever e analisar a associação entre a expansão de ESB/ESF por habitante e a diferença de taxas no uso de serviços odontológicos públicos nos municípios brasileiros entre 1999 e 2011.

\section{Metodologia}

\section{Delineamento}

Estudo ecológico longitudinal, em que as unidades de observação foram todos os municípios brasileiros. Foram selecionados dois anos: o ano inicial (linha de base) utilizado foi 1999 (anterior à Portaria no 1.444 de dezembro de 2000 3, que estabeleceu incentivo à incorporação de ESB nas ESF) e o ano final 2011. Com isso, pode-se avaliar o efeito da "intervenção natural" um ano antes e onze anos após a implantação das equipes de saúde bucal na ESF.

\section{Procedimento amostral}

Todos os municípios brasileiros foram incluídos na análise descritiva, totalizando 5.507 para o ano base e 5.565 para o ano final. Para as análises longitudinais, o tamanho amostral foi igual a 5.507, sendo excluídos os 58 municípios brasileiros criados após 1999. 
- Variáveis de interesse e fontes de dados

Todas as fontes de dados utilizadas podem ser acessadas no site do Departamento de Informática do SUS (DATASUS).

\section{- Variáveis sobre ESB (principal variável independente)}

Foram obtidas informações sobre o número de ESB/ESF nas modalidades 1, 2 e o total para cada município brasileiro em 2011. Após a obtenção desses valores, as ESB modalidade 1 (ESB1) e o total de equipes foram codificadas em quatro categorias: (a) zero equipes, (b) $>0$ a 1 equipe por 100 mil habitantes (maior que zero a um), (c) > 1 a 3 equipes por 100mil habitantes (maior que um a três), e (d) > 3 equipes por 100 mil habitantes (maior que três). As ESB modalidade 2 (ESB2) foram categorizadas em duas: sem e com ESB2. A fonte desses dados foi o Cadastro Nacional de Estabelecimentos de Saúde (CNES - cujos dados estão disponibilizados no site do DATASUS a partir de agosto de 2005).

\section{- Indicadores de estrutura dos serviços: variáveis relativas à oferta dos serviços de saúde}

Foram selecionados três grupos de variáveis: recursos humanos, físicos e financeiros. No grupo de variáveis de recursos humanos foram coletados (somente profissionais disponíveis para o SUS): (a) o número de cirurgiões dentistas (CD) (todas as especialidades); (b) número de auxiliares de saúde bucal (ASB); e (c) número de técnicos em saúde bucal (TSB). A fonte desses dados para o ano de 1999 foi a Pesquisa de Assistência Médico-Sanitária (PAMS); para o ano de 2011, foi utilizado o CNES.

No grupo de variáveis sobre recursos físicos (apenas em serviços disponíveis ao SUS), foram coletados: (a) número de equipes odontológicos; e (b) número de aparelhos de raios X odontológicos. Para ambos, as fontes e os anos foram os mesmos que para os recursos humanos (PAMS 1999 e CNES 2011).

No grupo de variáveis sobre recursos financeiros, foi obtido o valor em Reais (R\$) dos investimentos (despesas) totais per capita em saúde, cuja fonte foi o Sistema de Informações sobre Orçamentos Públicos em Saúde Pública (SIOPS), tanto para 1999 quanto para 2011.

\section{- Covariadas (características sociodemográficas)}

Foram coletados dados sobre variáveis sociodemográficas que em nível municipal poderiam estar associadas ao uso dos serviços. Com base nos dados censitários, oriundos do Instituto Brasileiro de Geografia e Estatística (IBGE), foram obtidos: (a) porte populacional; (b) percentual da população do sexo feminino; (c) percentual da população residente na zona urbana; (d) caracterização etária da população (0-14, 14-59 e 60 anos ou mais); e (e) PIB per capita (2000 e 2009 foram os anos mais próximos disponíveis). Com base nos dados da Agência Nacional de Saúde Suplementar (ANS), foi obtido também o percentual da população possuidora de planos privados de saúde odontológicos (disponível o do ano 2000, mas não o de 1999).

\section{- Indicadores de processo: variáveis relativas ao uso dos serviços de saúde}

Foram obtidos os seguintes dados do Sistema de Informações Ambulatoriais do SUS (SIA-SUS): (a) número de procedimentos coletivos (PC); (b) número de procedimentos preventivos (PP); (c) número de procedimentos odontológicos restauradores (PR); e (d) número de procedimentos de exodontia (PE).

Cada procedimento em saúde possui um código correspondente para registro nos sistemas de informações em saúde do Ministério da Saúde. Para o período de janeiro até dezembro de 1999, foram somados os códigos de 4 categorias de procedimentos odontológicos, conforme descrito por Celeste et al. 9 (PC, PP, PR e PE). Em janeiro de 2008, a tabela de códigos de procedimentos do SIA-SUS teve alteração de nomenclatura e códigos 23. Então, para o ano de 2011 (janeiro até dezembro) foram somados os códigos correspondentes de forma a manter a comparabilidade (a lista completa dos códigos pode ser obtida em contato com os autores).

\section{Construção dos indicadores}

As taxas anuais do indicador ESB/ESF foram calculadas por 10 e por $100 \mathrm{mil}$, as de recursos físicos e humanos, por $100 \mathrm{mil}$, e as de processo, por mil habitantes. Para os componentes de estrutura do ano final e procedimentos de 1999 e 2011, foi primeiramente calculada a média mensal (CNES e SIA-SUS fornecem os valores por mês) e posteriormente foi calculada a taxa. Quanto aos componentes de estrutura de 1999, a PAMS fornece apenas um valor correspondente ao ano, dispensando o cálculo da média anual antes de 
calcular a taxa. Para fins de análise, da taxa de 2011 foi subtraída a taxa de 1999, sendo um valor positivo considerado um aumento. Tal procedimento foi feito para as variáveis de desfecho e para as independentes.

Durante a edição de dados foram diagnosticados 148 municípios com valores extremos em variáveis de estrutura e de uso dos serviços e que refletiam potencialmente erros, sendo 77 no ano base e 71 no ano final. Todos estes valores foram excluídos. Análise de sensibilidade mostrou que tais exclusões não alteraram as medidas de associação observadas.

\section{Análise estatística}

A estatística descritiva foi realizada pela média, desvio padrão, intervalo de $95 \%$ de confiança (IC95\%) e percentual (Tabelas 1 e 2). Foram realizadas análises bivariadas de correlação entre as categorias de ESB/ESF e a quantidade de municípios que aumentaram as taxas de procedimentos pelo teste qui-quadrado (Tabela 2).

Análises por regressão múltipla foram realizadas utilizando-se como desfecho a variação do município (redução ou nenhum aumento = 0 ; aumento $=1$ ) em cada um dos quatro procedimentos descritos (PC, PP, PR, PE) no período entre 1999 e 2011. Calcularam-se as chances de um município ter aumento da produção ambulatorial por meio de modelos de regressão logística normal. A principal variável independente de interesse foi o número de ESB por 10 mil habitantes. Como variáveis de ajuste, foram utilizadas as variáveis de estrutura (recursos humanos, físicos e financeiros) e fatores sociodemográficos no ano de 1999 (baseline) e também a variação do município no período entre 1999 e 2011. Nos modelos ajustados, foi utilizada a técnica stepwise, sendo estipulado o valor de $\mathrm{p} \leq 0,01$ para entrada e p $<0,05$ para permanência no modelo multivariado. Foram apresentados odds ratios, IC95\% e valor de $\mathrm{p}$ (Tabela 3 ).

A elaboração do banco, edição e análise descritiva dos dados foi realizada com uso do programa estatístico R 2.14.2 (The R Foundation for Statistical Computing, Viena, Áustria; http:// www.r-project.org), enquanto para as análises de regressão foi utilizado o Stata 11.2 (StataCorp LP, College Station, Estados Unidos).

\section{Resultados}

Observou-se que em 2011, 85,6\% dos municípios brasileiros $(n=4.763)$ possuíam ESB/ESF, contando com uma taxa média mensal de 22,7 $(\mathrm{DP} \pm 15,2) \mathrm{ESB} / \mathrm{ESF}$ por 100 mil habitantes. Con- traditoriamente, de 1999 a 2011 a taxa do total de CD apresentou queda igual a 1,9 por $100 \mathrm{mil}$ habitantes $(3,4 \%)$, diminuindo de 30,5 para 28,6 , ainda que pouco mais da metade $(50,4 \%)$ dos municípios tenham aumentado sua cobertura populacional de CD. Em relação aos recursos humanos, observou-se aumento da sua cobertura populacional, com aumento respectivo em $72,8 \%$ e $17,1 \%$ dos municípios brasileiros (Tabela 1).

Em relação aos recursos físicos, as taxas médias de equipos e raio $\mathrm{X}$ odontológicos disponíveis ao SUS apresentaram aumento ao longo dos anos. Tal aumento representou 14,8 (75\%) equipos por 100 mil habitantes a mais, passando de 20,0 em 1999 para 34,8 em 2011, e 78,1\% dos municípios aumentaram suas taxas de equipos; e $2,9(66,7 \%)$ raio $\mathrm{X}$ a mais por $100 \mathrm{mil}$ habitantes, passando de 2,6 no ano base para 5,5 no ano final, e $34,2 \%$ dos municípios aumentaram a taxa desse componente da oferta. Quanto aos recursos financeiros, o investimento per capita em saúde médio realizado pelos municípios brasileiros era igual a $\mathrm{R} \$$ 83,70 em 1999 e $\mathrm{R} \$$ 445,40 em 2011, uma diferença de $\mathrm{R} \$ 361,70$, o que representa um expressivo aumento de $512 \%$. Todos os municípios brasileiros que existiam em 1999 aumentaram seu investimento per capita em saúde em 2011.

Ressalta-se que tanto os recursos humanos quanto físicos analisados aqui se referem aos serviços públicos em todos os níveis de atenção, e não somente à atenção básica (Tabela 1).

Em relação ao uso dos serviços de saúde bucal no ano base, a taxa média total de procedimentos odontológicos por mil habitantes nos municípios brasileiros foi igual a 86,5, e no ano final foi igual a 129,6, representando um aumento de 49,5\% (dados não disponibilizados em tabela). Todas as taxas de utilização de serviços apresentaram aumentos. As taxas de extrações foram as que apresentaram o maior aumento percentual no período $(74,1 \%)$, passando de 9,9 para 17,3 , seguidas dos procedimentos coletivos (50,1\%), que aumentaram de 43,8 para 65,7 , restaurações (48\%), que subiram de 17,1 para 25,3 , e procedimentos preventivos $(27,9 \%)$, que passaram de 15,7 para 20,1 por 10 mil habitantes (valores percentuais acima não estão disponibilizados em tabela). Os percentuais de municípios que aumentaram as taxas de PC, PP, PR e PE foram, respectivamente: $66,5 \%, 60,6 \%, 62,8 \%$ e $37,5 \%$ (Tabela 1).

Quanto à quantidade de municípios que aumentaram suas taxas de procedimentos odontológicos segundo a cobertura de ESB/ESF por habitante, as distribuições apresentaram-se diretamente proporcionais para as quatro taxas de procedimentos, nas três modalidades de ESB/ 
Descrição dos componentes da oferta e do uso de serviços odontológicos públicos e fatores sociodemográficos nos municípios brasileiros em 1999 e 2011.

\begin{tabular}{|c|c|c|c|c|c|c|c|c|}
\hline & \multicolumn{3}{|c|}{1999} & \multicolumn{3}{|c|}{2011} & \multirow[t]{2}{*}{ Incremento 1999-2011 } & \multirow{2}{*}{$\begin{array}{l}\% \text { municípios } \\
\text { com aumento }\end{array}$} \\
\hline & Média & $( \pm \mathrm{DP})$ & $\mathbf{N}$ & Média & $( \pm D P)$ & $\mathbf{N}$ & & \\
\hline \multicolumn{9}{|l|}{ Estrutura: fatores relacionados à oferta } \\
\hline \multicolumn{9}{|l|}{ dos serviços de saúde } \\
\hline ESB-ESF/100 mil habitantes & - & - & - & 22,7 & 15,2 & 5.565 & 22,7 & 85,6 \\
\hline CD/100 mil habitantes & 30,5 & 27,2 & 5.449 & 28,6 & 20,8 & 5.547 & $-1,9$ & 50,4 \\
\hline ASB/100 mil habitantes & 12,5 & 18,4 & 5.501 & 26,2 & 18,0 & 5.564 & 13,7 & 72,8 \\
\hline TSB/100 mil habitantes & 1,1 & 5,4 & 5.506 & 1,4 & 4,0 & 5.565 & 0,3 & 17,1 \\
\hline Equipos/100 mil habitantes & 20,0 & 21,0 & 5.496 & 34,8 & 19,8 & 5.550 & 14,8 & 78,1 \\
\hline Raio-X/100 mil habitantes & 2,6 & 8,4 & 5.506 & 5,5 & 11,5 & 5.564 & 2,9 & 34,2 \\
\hline Investimento per capita em saúde (R\$) & 83,7 & 48,5 & 5.336 & 445,4 & 196,0 & 5.279 & 361,7 & 100,0 \\
\hline \multicolumn{9}{|l|}{ Processo: fatores relacionados ao uso } \\
\hline \multicolumn{9}{|l|}{ dos serviços de saúde } \\
\hline $\mathrm{PC} / \mathrm{mil}$ habitantes & 43,8 & 77,3 & 5.507 & 65,7 & 131,0 & 5.547 & 20,9 & 66,5 \\
\hline $\mathrm{PP} / \mathrm{mil}$ habitantes & 15,7 & 34,6 & 5.507 & 20,1 & 117,7 & 5.564 & 4,4 & 60,6 \\
\hline PR/mil habitantes & 17,1 & 21,7 & 5.507 & 25,3 & 124,5 & 5.563 & 10,4 & 62,8 \\
\hline $\mathrm{PE} / \mathrm{mil}$ habitantes & 9,9 & 9,9 & 5.507 & 17,3 & 183,8 & 5.565 & 7,5 & 37,5 \\
\hline \multicolumn{9}{|l|}{ Fatores sociodemográficos } \\
\hline$\%$ Mulheres & 49,2 & 1,5 & 5.507 & 49,5 & 1,6 & 5.565 & 0,3 & 70,1 \\
\hline$\%$ Crianças/Jovens até 14 anos & 31,6 & 5,3 & 5.507 & 25,3 & 5,0 & 5.565 & $-0,6$ & 0,1 \\
\hline$\%$ Adultos (15-60 anos) & 58,9 & 4,2 & 5.507 & 62,6 & 3,4 & 5.565 & 0,4 & 97,1 \\
\hline$\%$ Idosos (> 60 anos) & 9,5 & 2,5 & 5.507 & 12,1 & 3,3 & 5.565 & 2,6 & 99,0 \\
\hline \% Zona urbana & 58,8 & 23,3 & 5.507 & 63,8 & 22,0 & 5.565 & 5,3 & 87,0 \\
\hline$\%$ Cobertura planos privados & 0,2 & 1,0 & 5.507 & 1,6 & 3,7 & 5.563 & 1,4 & 95,2 \\
\hline \multicolumn{9}{|l|}{ odontológicos } \\
\hline PIB municipal per capita (R\$) & $4.046,5$ & $4.645,2$ & 5.507 & $10.980,0$ & $12.150,7$ & 5.565 & 6.856 & 97,1 \\
\hline
\end{tabular}

ASB: auxiliares de saúde bucal; CD: cirurgião dentista; ESB: equipes de saúde bucal; ESF: Estratégia Saúde de Família; PC: procedimentos coletivos; PE: procedimentos de exodontia; PIB: Produto Interno Bruto; PP: procedimentos preventivos; PR: procedimentos odontológicos restauradores; TSB: técnicos de saúde bucal.

ESF (1, 2 e total). Exceção foi a relação entre ESB1 e PE, na qual se pôde observar que somente com uma cobertura > 3 ESB1 por 10 mil habitantes a frequência de municípios que aumentaram PE foi significativamente maior que aqueles que não possuíam ESB1. Apesar de não ter existido tendência de crescimento de municípios que aumentaram taxas de $\mathrm{PE}$ em relação à cobertura de ESB1 por 10mil habitantes, a associação dessa distribuição apresentou-se estatisticamente significativa ao teste qui-quadrado (valor de $\mathrm{p}$ $<0,01)$. Esse teste foi significativo para todos os demais desfechos em relação às coberturas de ESB1 e ESB total por 10mil habitantes. Em relação à cobertura de ESB2 por 10mil habitantes, apenas a tendência dos municípios que aumentaram taxas de PC não foi estatisticamente significativa (valor de $p=0,13$ ). De fato, a diferença entre aqueles sem e com ESB2 mostrou-se muito pequena, na ordem de 2,7 pontos percentuais (Tabela 2).

No modelo bruto de regressão logística, a cobertura de até 1 ESB/ESF por 10mil habitantes esteve associada a um aumento de 1,36 vez (IC95\%: 1,09-69) nas chances de os municípios aumentarem a sua taxa de PC, ao passo que a cobertura $>3 \mathrm{ESB} / \mathrm{ESF}$ por 10mil habitantes esteve relacionada a um aumento de 2,97 vezes (IC95\%: $1,66-2,38)$ nas chances de os municípios aumentarem sua taxa de PC de 1999 a 2011. No modelo ajustado, entretanto, apenas a presença de mais de 3 ESB/ESF foi significativa (OR = 1,61; IC95\%: 1,23-2,11) (Tabela 3). Resultados similares foram obtidos para os PR, PP e PE, isto é, houve redução na magnitude de associação no modelo ajustado, porém todos permaneceram estatisticamente significativos quando a presença de $>3 \mathrm{ESB} / \mathrm{ESF}$ esteve associada a maiores chances de o municí- 
Frequência de municípios brasileiros que aumentaram as taxas de procedimentos odontológicos entre 1999 e 2011, por modalidade de equipe de saúde bucal (ESB/10 mil habitantes).

\begin{tabular}{|c|c|c|c|c|c|c|c|c|}
\hline \multirow{2}{*}{$\begin{array}{l}\text { Modalidade/Equipes/10 mil } \\
\text { habitantes }\end{array}$} & \multicolumn{2}{|c|}{ Aumentaram PC } & \multicolumn{2}{|c|}{ Aumentaram PP } & \multicolumn{2}{|c|}{ Aumentaram PR } & \multicolumn{2}{|c|}{ Aumentaram PE } \\
\hline & $\%$ & n & $\%$ & $n$ & $\%$ & $n$ & $\%$ & $n$ \\
\hline Total & 66,5 & 3.651 & 60,0 & 3.306 & 62,8 & 3.458 & 37,5 & 2.066 \\
\hline \multicolumn{9}{|l|}{ ESB modalidade 1} \\
\hline 0 & 53,5 & 424 & 39,5 & 313 & 46,8 & 371 & 26,4 & 209 \\
\hline$>0-1$ & 58,7 & 467 & 50,9 & 406 & 49,9 & 398 & 27,1 & 216 \\
\hline$>1-3$ & 67,6 & 1.616 & 63,1 & 1.514 & 65,7 & 1.570 & 24,6 & 950 \\
\hline$>3$ & 75,7 & 1.114 & 72,8 & 1.103 & 73,9 & 1.119 & 45,6 & 691 \\
\hline \multicolumn{9}{|l|}{ ESB modalidade 2} \\
\hline Sem & 64,2 & 507 & 52,0 & 412 & 58,3 & 462 & 33,3 & 264 \\
\hline Com & 66,9 & 3.144 & 62,0 & 2.924 & 63,6 & 2.996 & 38,2 & 1.802 \\
\hline \multicolumn{9}{|l|}{ ESB total } \\
\hline 0 & 50,3 & 296 & 38,1 & 224 & 43,5 & 256 & 23,6 & 139 \\
\hline$>0-1$ & 57,9 & 422 & 50,1 & 336 & 48,0 & 351 & 26,9 & 197 \\
\hline$>1-3$ & 66,8 & 1.613 & 61,5 & 1.492 & 64,0 & 1.567 & 38,8 & 940 \\
\hline$>3$ & 75,1 & 1.320 & 71,2 & 1.254 & 72,9 & 1.284 & 44,8 & 790 \\
\hline
\end{tabular}

PC: procedimentos coletivos; PE: procedimentos de exodontia; PP: procedimentos preventivos; PR: procedimentos odontológicos restauradores Nota: o valor de p do qui-quadrado foi estatisticamente significativo $(>0,05)$ para todas as associações, exceto "aumentaram PC" $x$ "ESB modalidade 2 " $(=0,13)$.

pio aumentar PR (OR = 2,07; IC95\%: 1,58-2,71), $\mathrm{PP}(\mathrm{OR}=2,05$; IC95\%: 1,56-2,69), e PE (OR = 1,53; IC95\%: 1,19-1,97).

É importante também levar em consideração que nos modelos ajustados, as variáveis de ajuste foram diferentes para cada desfecho. Isso se deu porque a utilização do método stepwise manteve variáveis distintas. Como o número de covariadas mostrou-se extenso, resultados pormenorizados não foram apresentados.

\section{Discussão}

O presente trabalho tem como principal resultado que a incorporação de equipes de saúde bucal na Saúde da Família esteve associada a mais chances de aumentar taxas de uso de serviços (indicadores de processo), representadas por variáveis de produtividade. Esse efeito associado ao acréscimo de ESB/ESF permaneceu após controle por fatores sociodemográficos e por fatores relacionados à variação dos recursos físicos, humanos e financeiros (indicadores de estrutura).

Foi analisada em nosso estudo a relação entre mudanças nos indicadores de estrutura e mudanças nos indicadores de processo nos serviços odontológicos públicos em todos os municípios brasileiros, entre 1999 e 2011. A utilização de um conjunto de indicadores, ao invés de um indicador único, vem ao encontro das metodologias utilizadas para avaliação de sistemas e serviços de saúde em países como os Estados Unidos e a Inglaterra 13. No Brasil, o Ministério da Saúde também utiliza conjuntos de indicadores para monitorar e avaliar as ações em saúde, a exemplo do Pacto de Indicadores da Atenção Básica e dos Pactos pela Vida, em Defesa do SUS e de Gestão ${ }^{23}$, reconhecendo o perfil complexo da realidade a ser avaliada. Salienta-se aqui que este estudo não se propõe a realizar uma avaliação de qualidade propriamente dita 21 . Acredita-se que a expansão de indicadores de estrutura podem influenciar os indicadores de processos, aumentando ou diminuindo as taxas de utilização. Contudo, o incremento da oferta (p. ex.: aumento na taxa de $\mathrm{CD} /$ habitante), por si só, não garante aumento no uso nem na melhoria da qualidade dos serviços, tampouco nos níveis de saúde da população 13,24,25.

É possível que o acréscimo verificado na produtividade seja devido ao modelo de atenção adotado na ESF, já que o efeito permaneceu após o controle tanto da estrutura existente em 1999 como pela expansão até 2011. Experiências bem-sucedidas da prática das ESB/ESF estão documentadas e podem ajudar a explicar porque esse modelo foi associado a uma maior produ- 
Modelos de regressão logística stepwise (bruto e ajustado) entre presença de ESB/ESF e aumento de taxas de procedimentos odontológicos entre 1999-2011, em 5.020 municípios brasileiros.

\begin{tabular}{|c|c|c|c|c|c|c|}
\hline \multirow[t]{2}{*}{ ESB/10 mil habitantes } & \multicolumn{3}{|c|}{ Modelo bruto } & \multicolumn{3}{|c|}{ Modelo ajustado } \\
\hline & OR & IC95\% & Valor de $p$ & OR & IC95\% & Valor de $p$ \\
\hline \multicolumn{7}{|l|}{$P C$ * } \\
\hline 0 & 1,00 & & $<0,01$ & 1,00 & & $<0,01$ \\
\hline$>0-1$ & 1,36 & $1,09-1,69$ & & 0,90 & $0,70-1,16$ & \\
\hline$>1-3$ & 1,99 & $1,66-2,38$ & & 1,09 & $0,85-1,38$ & \\
\hline$>3$ & 2,97 & $2,45-3,61$ & & 1,61 & $1,23-2,11$ & \\
\hline \multicolumn{7}{|l|}{$\mathrm{PP} * \star$} \\
\hline 0 & 1,00 & & $<0,01$ & 1,00 & & $<0,01$ \\
\hline$>0-1$ & 1,63 & $1,31-2,03$ & & 1,10 & $0,85-1,42$ & \\
\hline$>1-3$ & 2,60 & $2,16-3,13$ & & 1,46 & $1,14-1,87$ & \\
\hline$>3$ & 4,01 & $3,30-4,88$ & & 2,05 & $1,56-2,69$ & \\
\hline \multicolumn{7}{|l|}{$\mathrm{PR} * \star \star$} \\
\hline 0 & 1,00 & & $<0,01$ & 1,00 & & $<0,01$ \\
\hline$>0-1$ & 1,20 & $0,96-1,49$ & & 0,76 & $0,59-0,97$ & \\
\hline$>1-3$ & 2,37 & $1,97-2,85$ & & 1,40 & $1,10-1,78$ & \\
\hline$>3$ & 3,48 & $2,87-4,23$ & & 2,07 & $1,58-2,71$ & \\
\hline \multicolumn{7}{|l|}{ PE \# } \\
\hline 0 & 1,00 & & $<0,01$ & 1,00 & & $<0,01$ \\
\hline$>0-1$ & 1,19 & $0,93-1,53$ & & 0,83 & $0,62-1,10$ & \\
\hline$>1-3$ & 2,04 & $1,66-2,51$ & & 1,30 & $1,03-1,64$ & \\
\hline$>3$ & 2,62 & $2,12-3,24$ & & 1,53 & $1,19-1,97$ & \\
\hline
\end{tabular}

ASB: auxiliares de saúde bucal; CD: cirurgião dentista; ESB: equipes de saúde bucal; ESF: Estratégia Saúde de Família; IC95\%: intervalo de 95\% de confiança; OR: odds ratio; PC: procedimentos coletivos; PE: procedimentos de exodontia; PP: procedimentos preventivos; PR: procedimentos odontológicos restauradores; TSB: técnicos de saúde bucal.

* Permaneceram no modelo final: \% de população feminina, \% de população urbana e \% de população 0-14 anos, incremento de ASB e incremento de TSB;

** Permaneceram no modelo final: \% de população feminina, \% população de urbana, \% de 0-14, \% de 15-59 anos, investimento per capita em saúde, CD, ASB, TSB e equipes;

*** Permaneceram no modelo final: \% de população de 0-14 e de população 15-59 anos, cobertura de plano odontológico، investimento per capita em saúde, CD, ASB e raio $X$;

\# Permaneceram no modelo final: \% de população feminina, \% de população urbana, \% de 0-14, \% de 15-59 anos, investimento per capita em saúde, CD e equipes.

tividade. Dentre esses aspectos, são destacados: a delimitação da clientela possibilitando melhor acompanhamento; a cobertura populacional de acordo com o estipulado pelo Ministério da Saúde; a recepção favorável e satisfação por parte do usuário; a existência de relações humanizadoras e vínculo entre trabalhadores; incorporação de CD em tempo integral; a capacitação do CD na área de saúde pública e sua boa integração com a equipe e usuários 8,11,25,26,27. Entretanto, tais experiências também podem ser encontradas em unidades básicas de saúde tradicionais (sem ESF), o que pode explicar o aumento da produção ambulatorial em municípios sem ESB. Especula-se que eles já estariam trabalhando numa lógica mais distante dos modelos assistenciais tradicionais em saúde bucal 2 . Salienta-se que a literatura sobre o processo de trabalho das ESB/ ESF é escassa e também enumera aspectos negativos, como a dificuldade de mudança do modelo assistencial tradicional 12 . De fato, de $24,9 \%$ a $55,2 \%$ (dependendo do grupo de procedimentos) dos municípios com mais de três ESB/ESF por 10 mil habitantes apresentaram diminuição na sua taxa de uso (Tabela 2).

Destacamos, porém, outros fatores que podem explicar o aumento no uso dos serviços mediante o incremento da cobertura de ESB/ ESF por 10 mil habitantes. O primeiro deles diz respeito ao atendimento à demanda reprimida 
em regiões que anteriormente não possuíam oferta de serviço de saúde bucal à população. $\mathrm{O}$ segundo fator corresponde à carga-horária dos profissionais da ESF, que é de 40 horas semanais, em oposição às 20 horas dos profissionais das UBS tradicionais. Estudos estimaram que entre $85,8 \%$ e $91 \%$ dos profissionais das ESB/ESF trabalham em regime de 40 horas semanais 26,29 . Um terceiro fator estaria relacionado ao tempo de implantação das ESB/ESF, considerando as possíveis limitações inerentes a essa variável, por exemplo: tempo necessário para que uma ESB/ ESF esteja apta a trabalhar satisfatoriamente e a intermitência de manutenção da implantação de muitas delas.

Uma das características da saúde bucal na ESF é a presença de auxiliares e técnicos em saúde bucal. Estudos internacionais 30,31 têm sugerido que a inclusão desses profissionais nas equipes de saúde podem aumentar o acesso e a produtividade e reduzir as disparidades em saúde bucal, assim como diminuir os custos da atenção. Segundo Warmling ${ }^{32}$, a identidade desses profissionais vinculados ao SUS em termos de competência profissional está ligada às ações coletivas. Além disso, as atribuições das competências de atendimento clínico e de organização do ambiente de trabalho são as que mais conferem identidade ao trabalho dessas categorias profissionais. Segundo os resultados do nosso estudo, o aumento nas taxas de ASB e TSB não anulam a associação entre a expansão da cobertura populacional de ESB/ESF e o aumento da produtividade. Mais importante que a quantidade de profissionais, então, seria o modelo e contexto em que se dá o seu trabalho.

Taxas de procedimentos odontológicos são medidas frequentemente usadas para expressar acesso em saúde bucal, refletindo, na verdade, o uso dos serviços 33. Medidas de uso, portanto, são uma medida mista de acesso e demanda (i.e., necessidade percebida), visto que representam o acesso concretizado pelos usuários que demandaram esses serviços 34; o que Andersen 35 chamou de acesso realizado. Neste estudo, a taxa total de procedimentos odontológicos por mil habitantes apresentou aumento de quase $50 \%$ de 1999 a 2011, ilustrando o aumento do acesso da população aos serviços de saúde bucal nos municípios brasileiros. Tal descoberta está em consonância com achados de tendências entre 2000 e 2007, que mostraram um aumento de 6,9\% no total de procedimentos e $15,9 \%$ para procedimentos coletivos ${ }^{9}$. Por outro lado, o estudo citado também relatou uma redução de procedimentos preventivos, exodontias e restaurações 9 . Outro estudo, em Pernambuco, analisou indicadores longitudinalmente, mostrando $327,1 \%$ de aumento de ESB1 e de 125,9\% para ESB2, com um aumento de $19,2 \%$ na produção de procedimentos odontológicos ambulatoriais básicos no SUS 11. De 1999 a 2006, no Município de Fortaleza, Ceará, a utilização de serviços odontológicos foi a única dimensão que apresentou piora entre cinco dimensões da gestão da atenção básica em saúde bucal avaliadas, em razão da queda no indicador primeira consulta odontológica e apesar da melhora na proporção de procedimentos odontológicos especializados em relação às ações individuais de Atenção Básica 36. Pereira et al. 37 verificaram que os indicadores cobertura de exodontia, tratamento restaurador e dor de dente demonstraram efeito negativo ou sem efeito na maioria dos municípios pesquisados, incluindo aqueles sem saúde bucal na ESF ou sem cobertura populacional. Por outro lado, acesso a ações preventivas e coletivas apontaram um efeito positivo nos municípios com ESB na ESF.

O presente estudo apresenta alguns pontos fortes, mas também limitações. Foram utilizados dados de sistemas oficiais de informação, com abrangência de todos os municípios brasileiros. Com isso, exclui-se a possibilidade de vieses de seleção e perda de representatividade. O delineamento longitudinal utilizado permite inferir alterações nas variáveis ao longo tempo, mas não garante o estabelecimento de relação causal. A não utilização de séria histórica (estudo de adequação), contudo, não invalida as análises realizadas, uma vez que a presente investigação caracteriza-se como um estudo de plausibilidade 38 . Ou seja, foi utilizado um desenho observacional com grupo controle (ausência de ESB/ESF) a fim de realizar potenciais inferências causais, após exclusão de hipóteses alternativas. Considerou-se tal abordagem apropriada para uma avaliação de larga escala de provável cadeia causal complexa como a realizada (ainda que o uso de serviços seja considerado um resultado intermediário), além de apresentar validades interna e externa.

Quanto aos sistemas de informação, a qualidade de alguns deles está aquém do desejável, e a possibilidade de erros de notificação é possível. Pode existir, por exemplo, subnotificação e erro de notificação em taxas de procedimentos específicos. Supernotificação, por sua vez, pode ser esperada em municípios que gratificam os profissionais por desempenho e naqueles em que existe supervisão sobre metas de produtividade, a exemplo de serviços terceirizados. Outra limitação relacionada às fontes de dados refere-se às diferentes bases utilizadas para as variáveis de estrutura (PAMS para o ano de 1999 e CNES para 2011), o que pode explicar a redução na taxa de CD verificada neste estudo. 
O tema pesquisado no presente estudo é de extrema importância e apresenta respostas úteis a gestores e pesquisadores. A principal delas é que o aumento no uso dos serviços odontológicos públicos no Brasil mostrou-se associado ao aumento nas taxas de equipes de saúde bucal da ESF, independente do crescimento da estrutura. Considerando que muitos municípios sem ESB/ ESF também aumentaram sua produção odontológica ambulatorial, os entraves existentes no sistema e as limitações do presente estudo, ainda é necessário conhecer melhor os processos de trabalho em diferentes cenários, quem se beneficia dessa expansão 19,20 bem como a influência de fatores individuais e características dos profissionais e do processo de trabalho no uso dos serviços. Este estudo conclui, então, que a incorporação de ESB à ESF parece ser estratégia mais efetiva para o aumento de indicadores de utilização de serviços odontológicos. Por fim, com o intuito de reforçar (ou refutar) tal afirmativa, faz-se necessária a realização de estudos de série histórica 25,38, além de ensaios randomizados e controlados em saúde pública 39 .

\section{Resumen}

Analizar la asociación entre la cobertura de equipos de salud bucal en la Estrategia Salud de la Familia (ESF) y el cambio de tasa de uso de servicios dentales en los municipios brasileños en 1999 y 2011. La muestra estuvo constituida por los 5.507 municipios durante los dos años. Se utilizaron datos de sistemas de información oficiales y se procedió a gestionarlos mediante regresión logística para modelar el aumento en las tasas. Se observó que el $85 \%$ de los municipios tenían equipos de salud bucal en 2011 y hubo aumentos en las tasas de los recursos físicos, humanos y financieros. La producción dental aumentó un 49,5\% interanualmente. Los municipios que han incorporado $>3$ equipos de salud bucal/10 mil habitantes tenían más probabilidades de aumentar las tasas de los procedimientos colectivos $(O R=1,61$; IC95\%: 1.23-2,11), preventivos $(O R=$ 2,05; IC95\%: 1.56-2,69), restauraciones $(O R=2,07$ IC95\%: 1,58-2,71) y extracciones $(O R=1,53$; IC95\%: 1,19-1,97), después de ajustar por factores sociodemográficos y la variación de recursos físicos, financieros y humanos. La incorporación de equipos de salud bucal parece más eficiente para aumentar el acceso de la población.

Servicios de Salud Dental; Atención Primaria de Salud; Accesibilidad a los Servicios de Salud; Políticas Públicas de Salud

\section{Colaboradores}

G. T. Corrêa participou da concepção, coleta, análise dos dados e redação do manuscrito. R. K. Celeste participou da concepção do estudo, análise dos dados, interpretação dos resultados e revisão do manuscrito.

\section{Agradecimentos}

Os autores agradecem aos revisores anônimos pelas suas contribuições ao aprimoramento da versão inicial deste artigo. Os autores agradecem também aos professores doutores: Fernando Neves Hugo, Cristine Warmling e Maria Beatriz Junqueira de Carmargo, pelas suas colaborações no aprimoramento do artigo, ainda na sua versão que compôe resultados de dissertação de mestrado. 


\section{Referências}

1. Pucca Jr. GA, Costa JF, Chagas LD, Sivestre RM Oral health policies in Brazil. Braz Oral Res 2009; 23 Suppl 1:9-16.

2. Chaves SC. Oral health in Brazil: the challenges for dental health care models. Braz Oral Res 2012; 26 Suppl 1:71-80.

3. Ministério da Saúde. Portaria no 1.444, de 28 de dezembro de 2000. Estabelece incentivo financeiro para a reorganização da atenção à saúde bucal prestada nos municípios por meio do Programa de Saúde da Família. Diário Oficial da União 2000; 29 dez.

4. Ministério da Saúde. Portaria GM/MS no 673, de 3 de junho de 2003. Atualiza e revê o incentivo financeiro às Ações de Saúde Bucal, no âmbito do Programa de Saúde da Família, parte integrante do Piso de Atenção Básica - PAB. Diário Oficial da União 2000; 4 jun.

5. Coordenação Nacional de Saúde Bucal, Departamento de Atenção Básica, Secretaria de Atenção à Saúde, Ministério da Saúde. Diretrizes da Política Nacional de Saúde Bucal. Brasília: Ministério da Saúde; 2004

6. Silveira Filho AD. A saúde bucal no PSF: o desafio de mudar a prática. Informe PSF 2002; Dezembro:36-43.

7. Junqueira SR, Pannuti CM, Rode SM. Oral health in Brazil. Part I: public oral health policies. Braz Oral Res 2008; 22 Suppl 1:8-17.

8. Pinheiro R, Torres TZG. Uso de serviços odontológicos entre os Estados do Brasil. Ciênc Saúde Coletiva 2006; 11:999-1010.

9. Celeste RK, Vital JF, Junger WL, Reichenheim ME. Séries de procedimentos odontológicos realizadas nos serviços públicos brasileiros, 1994-2007. Ciênc Saúde Coletiva 2011; 16:4523-32.

10. Pereira CR, Patricio AA, Araujo FA, Lucena EE, Lima KC, Roncalli AG. Impacto da Estratégia Saúde da Família com equipe de saúde bucal sobre a utilização de serviços odontológicos. Cad Saúde Pública 2009; 25:985-96.

11. Silva SF, Martelli PJL, Sa DA, Cabral AP, Pimentel FC, Monteiro IS, et al. Análise do avanço das equipes de saúde bucal inseridas na Estratégia Saúde da Família em Pernambuco, região Nordeste, Brasil, 2002 a 2005. Ciênc Saúde Coletiva 2011; 16:211-20.

12. Soares FF, Figueiredo CR, Borges NC, Jordao RA, Freire MC. Atuação da equipe de saúde bucal na estratégia saúde da família: análise dos estudos publicados no período 2001-2008. Ciênc Saúde Coletiva 2011; 16:3169-80.

13. Campbell SM, Roland MO, Buetow SA. Defining quality of care. Soc Sci Med 2000; 51:1611-25.

14. Hjern A, Grindefjord M, Sundberg H, Rosen M. Social inequality in oral health and use of dental care in Sweden. Community Dent Oral Epidemiol 2001; 29:167-74.

15. Hughes DC, Duderstadt KG, Soobader MP, Newacheck PW. Disparities in children's use of oral health services. Public Health Rep 2005; 120:455-62.
16. Stahlnacke K, Soderfeldt B, Unell L, Halling A, Axtelius B. Changes over 5 years in utilization of dental care by a Swedish age cohort. Community Dent Oral Epidemiol 2005; 33:64-73.

17. Ahacic K, Thorslund M. Changes in dental status and dental care utilization in the Swedish population over three decades: age, period, or cohort effects? Community Dent Oral Epidemiol 2008; 36:118-27.

18. Celeste RK, Nadanovsky P, Fritzell J. Trends in socioeconomic disparities in the utilization of dental care in Brazil and Sweden. Scand J Public Health 2011; 39:640-8.

19. Wanyonyi KL, Radford DR, Gallagher JE. The relationship between access to and use of dental services following expansion of a primary care service to embrace dental team training. Public Health 2013; 127:1028-33.

20. Celeste RK, Nadanovsky P. How much of the income inequality effect can be explained by public policy? Evidence from oral health in Brazil. Health Policy 2010; 97:250-8.

21. Donabedian A. The quality of care. How can it be assessed? JAMA 1988; 260:1743-8.

22. Donabedian A. Evaluating the quality of medical care. Milbank Mem Fund Q 1966; 44(3 Suppl): 166-206.

23. Departamento de Apoio à Gestão Descentralizada, Secretaria Executiva, Ministério da Saúde. Pactuação unificada de indicadores: avaliação 2007. Brasília: Editora MS; 2010. (Série G. Estatística e Informação em Saúde). (Série Pactos pela Saúde 2006; v. 11).

24. Donabedian A. An introduction to quality assurance in health care. New York: Oxford University Press; 2003.

25. Celeste RK, Nadanovsky P, Leon AP. Associação entre procedimentos preventivos no serviço público de odontologia e a prevalência de cárie dentária. Rev Saúde Pública 2007; 41:830-8.

26. Baldani MH, Fadel CB, Possamai T, Queiroz MG. A inclusão da odontologia no Programa Saúde da Família no Estado do Paraná, Brasil. Cad Saúde Pública 2005; 21:1026-35.

27. Emmi DT, Barroso RF. Avaliação das ações de saúde bucal no Programa Saúde da Família no distrito de Mosqueiro, Pará. Ciênc Saúde Coletiva 2008; 13:35-41.

28. Lourenco EC, Silva AC, Meneghin MC, Pereira AC. A inserção de equipes de saúde bucal no Programa Saúde da Família no Estado de Minas Gerais. Ciênc Saúde Coletiva 2009; 14 Suppl 1:1367-77.

29. Carvalho CL. Caracterização dos recursos humanos nos serviços públicos municipais de saúde bucal no Brasil. Relatório Técnico. Belo Horizonte: Observatório de Recursos Humanos em Saúde/ Núcleo de Educação em Saúde Coletiva; 2006. 
30. Bailit HL, Beazoglou TJ, DeVitto J, McGowan T, Myne-Joslin V. Impact of dental therapists on productivity and finances: I. Literature review. J Dent Educ 2012; 76:1061-7.

31. Nash DA. Adding dental therapists to the health care team to improve access to oral health care for children. Acad Pediatr 2009; 9:446-51.

32. Warmling CM. Identidade e prática do cirurgião-dentista. Pro-odonto Prevenção 2014; 3:31-52.

33. Petersen PE, Holst D. Utilization of dental health services. In: Cohen L, Gift H, editors. Disease prevention and oral health promotion: socio-dental sciences in action. Copenhagen: Munksgaard; 1995. p. 341-86.

34. Travassos C, Martins M. Uma revisão sobre os conceitos de acesso e utilização de serviços de saúde. Cad Saúde Pública 2004; 20 Suppl 2:S190-8.

35. Andersen RM. Revisiting the behavioral model and access to medical care: does it matter? J Health Soc Behav 1995; 36:1-10.
36. Lessa CFM, Vettore MV. Primary health care management in oral health in Fortaleza, Ceará, between 1999 and 2006. Saúde Soc 2010; 19:547-56.

37. Pereira CR, Roncalli AG, Cangussu MC, Noro LR, Patricio AA, Lima KC. Impacto da Estratégia Saúde da Família sobre indicadores de saúde bucal: análise em municípios do Nordeste brasileiro com mais de 100 mil habitantes. Cad Saúde Pública 2012; 28:449-62.

38. Victora CG, Habicht JP, Bryce J. Evidence-based public health: moving beyond randomized trials Am J Public Health 2004; 94:400-5.

39. Macintyre S. Good intentions and received wisdom are not good enough: the need for controlled trials in public health. J Epidemiol Community Health 2010; 65:564-7.

Recebido em 07/Jan/2015

Versão final reapresentada em 08/Mai/2015

Aprovado em 03/Jun/2015 